PROCEEDINGS OF THE AMERICAN MATHEMATICAL SOCIETY

Volume 124, Number 5, May 1996

\title{
THE STRUCTURE OF MEASURABLE MAPPINGS WITH VALUES IN LOCALLY CONVEX SPACES
}

\author{
JUN KAWABE
}

(Communicated by Palle E. T. Jorgensen)

\begin{abstract}
The purpose of this paper is to show that a theorem of A. Wisniewski remains valid without the approximation property.
\end{abstract}

\section{INTRODUCTION}

Let $X$ and $Y$ be Hausdorff topological spaces. Denote by $\mathcal{B}(X)$ and $\mathcal{B}(Y)$ respectively the Borel $\sigma$-algebras on these spaces. Let $\mu$ be a finite Borel measure on $X$. By $\mathcal{B}_{\mu}(X)$ we denote the completion of the $\sigma$-algebra $\mathcal{B}(X)$ with respect to the measure $\mu$. A mapping $f$ from $X$ into $Y$ is said to be $\mu$-measurable if it is measurable with respect to $\left(\mathcal{B}_{\mu}(X), \mathcal{B}(Y)\right)$. Recall that a Suslin space is a Hausdorff topological space which is the continuous image of some Polish space. It is well known but not easy to prove that every finite Borel measure on a Suslin space is Radon, and hence it is tight (see, e.g., [4, Theorem 10 of Chapter II in Part I]).

In [5], Wisniewski extended a theorem of Gihman and Skorohod [2, p. 544] which clarifies the connection between continuous and $\mu$-measurable mappings on Hilbert spaces. Namely, he proved that if $\mu$ is a finite Borel measure on a metric space $X$, and if $Y$ is a separable Banach space with the approximation property, then every $\mu$-measurable mapping $f$ from $X$ into $Y$ is the limit of a sequence of continuous mappings with respect to $\mu$-almost everywhere convergence.

In this paper, we show that the above result remains valid in the case that $Y$ is a Suslin locally convex topological linear space which does not necessarily have the approximation property. Consequently it turns out that Theorem 2 of [5] remains valid without the approximation property.

\section{MAin RESUlts}

Theorem 1. Let $\mu$ be a finite Borel measure on a metric space $X$, and let $Y$ be a locally convex Hausdorff topological linear space. Assume that every finite Borel measure on $Y$ is tight (this is satisfied, for instance, if $Y$ is Suslin). If $f$ is a $\mu$ measurable mapping from $X$ into $Y$, then there exists a sequence $\left\{f_{n}\right\}$ of continuous mappings from $X$ into $Y$ such that $f_{n} \rightarrow f \mu$-a.e.

Received by the editors October 25, 1994.

1991 Mathematics Subject Classification. Primary 28C15, 60B05; Secondary 28A20, 28C20, 60B11.

Key words and phrases. $\mu$-measurable mappings, continuous mappings, Suslin spaces, Banach spaces, Fréchet spaces, nuclear spaces, locally convex spaces, approximation property.

(C)1996 American Mathematical Society 
Proof. To prove the theorem it suffices to show that for any $\varepsilon>0$ and any closed neighborhood $U$ of the origin of $Y$, there exists a continuous mapping $g: X \rightarrow Y$ such that

$$
\mu\{x: f(x)-g(x) \notin U\}<\varepsilon .
$$

Since $Y$ is locally convex, we may assume that $U$ is absorbent and absolutely convex (see, e.g., [3, p. 12]). Put $\nu(B)=\mu\left(f^{-1}(B)\right)$ for every $B \in \mathcal{B}(Y)$. Then $\nu$ is a finite Borel measure on $Y$, and hence it is tight by the assumption. Thus we can find a compact subset $K$ of $Y$ such that $\nu(Y-K)<\varepsilon / 2$. Put $K^{\prime}=f^{-1}(K)$. Then we have

$$
\mu\left(X-K^{\prime}\right)<\frac{\varepsilon}{2}
$$

Since $K$ is compact, there exist $y_{1}, y_{2}, \cdots, y_{m} \in K$ such that

$$
K \subset\left(y_{1}+\frac{1}{2} U\right) \cup\left(y_{2}+\frac{1}{2} U\right) \cup \cdots \cup\left(y_{m}+\frac{1}{2} U\right) .
$$

Consider sets $B_{1}=y_{1}+\frac{1}{2} U, \cdots, B_{i}=\left\{\bigcup_{k=1}^{i-1}\left(y_{k}+\frac{1}{2} U\right)\right\}^{c} \cap\left(y_{i}+\frac{1}{2} U\right), \cdots$, $B_{m}=\left\{\bigcup_{k=1}^{m-1}\left(y_{k}+\frac{1}{2} U\right)\right\}^{c} \cap\left(y_{m}+\frac{1}{2} U\right)$. Then the $B_{i}$ 's are Borel subsets of $Y$ with $K \subset \bigcup_{i=1}^{m} B_{i}$ and $B_{i} \cap B_{j}=\emptyset$ if $i \neq j$. Hence

$$
f(x)-\sum_{i=1}^{m} 1_{B_{i}}(f(x)) y_{i} \in \frac{1}{2} U \text { for all } x \in K^{\prime}
$$

where $1_{B}$ denotes the indicator function of a set $B$. Consequently, using (1), we infer that

$$
\mu\left\{x: f(x)-\sum_{i=1}^{m} 1_{B_{i}}(f(x)) y_{i} \notin \frac{1}{2} U\right\} \leq \mu\left(X-K^{\prime}\right) \leq \frac{\varepsilon}{2} .
$$

For every $i=1,2, \cdots, m$, we put $f_{i}(x)=1_{B_{i}}(f(x))$ for all $x \in X$. Then the $f_{i}$ 's are $\mu$-measurable mapping from $X$ into the real line $R$. On the other hand, since $U$ is absorbent, there exists $\lambda>0$ such that $y_{i} \in \lambda U$ for all $i=1,2, \cdots, m$. Therefore, in view of Theorem 1 of [5], for every $i=1,2, \cdots, m$, we can find continuous mappings $g_{i}$ from $X$ into $R$ such that

$$
\mu\left\{x:\left|f_{i}(x)-g_{i}(x)\right|>\frac{1}{2 m \lambda}\right\}<\frac{\varepsilon}{2 m} .
$$

Put $g(x)=\sum_{i=1}^{m} g_{i}(x) y_{i}$ for every $x \in X$. Then $g$ is a continuous mapping from $X$ into $Y$. Moreover, from (2) and (3), together with the fact that $U$ is convex, we 
have

$$
\begin{aligned}
\mu\{x: & f(x)-g(x) \notin U\}=\mu\left\{x: f(x)-\sum_{i=1}^{m} g_{i}(x) y_{i} \notin U\right\} \\
\leq \mu & \left\{x: f(x)-\sum_{i=1}^{m} f_{i}(x) y_{i} \notin \frac{1}{2} U\right\} \\
& +\mu\left\{x: \sum_{i=1}^{m} f_{i}(x) y_{i}-\sum_{i=1}^{m} g_{i}(x) y_{i} \notin \frac{1}{2} U\right\} \\
\leq & \frac{\varepsilon}{2}+\sum_{i=1}^{m} \mu\left\{x:\left|f_{i}(x)-g_{i}(x)\right|>\frac{1}{2 m \lambda}\right\} \\
\leq & \varepsilon / 2+m \cdot \varepsilon /(2 m)=\varepsilon .
\end{aligned}
$$

This completes the proof of the theorem.

Remark. We can show that Theorem 1 also holds in the case that $X$ is a normal space and $\mu$ is a finite Borel measure on $X$ satisfying the condition that $\mu(B)=$ $\sup \{\mu(F): F \subset B$ and $F$ is a closed subset of $X\}$ for all $B \in \mathcal{B}(X)$.

Corollary. In the following cases, Theorem 1 holds:

(a) $Y$ is a separable Banach space.

(b) $Y$ is a separable Fréchet space.

(c) $Y$ is the strict inductive limit of an increasing sequence of separable Fréchet spaces.

(d) $Y$ is the strong dual of a nuclear Fréchet space.

(e) $Y$ is the strong dual of the strict inductive limit of an increasing sequence of nuclear Fréchet spaces.

Proof. (a)-(e) are Suslin (in fact, Lusin) locally convex topological linear spaces by [1, Theorem I.5.1].

\section{REFERENCES}

[1] X. Fernique, Processus linéaires, processus généralisés Ann. Inst. Fourier (Grenoble) 17 (1967), 1-92. MR 36:4628

[2] I. I. Gihman and A. V. Skorohod, The theory of stochastic processes. I, Springer-Verlag, Berlin, Heidelberg and New York, 1974. MR 49:11603

[3] A. P. Robertson and W. Robertson, Topological vector spaces, Cambridge Univ. Press, 1964. MR 28:5318

[4] L. Schwartz, Radon measures on arbitrary topological spaces and cylindrical measures, Oxford Univ. Press, 1973. MR 54:14030

[5] A. Wisniewski, The structure of measurable mappings on metric spaces, Proc. Amer. Math. Soc. 122 (1994), 147-150. MR 94k:28006

Department of Mathematics, Faculty of Engineering, Shinshu University, Wakasato, NAGANO 380, JAPAN

E-mail address: jkawabe@gipwc.shinshu-u.ac.jp 